\title{
Complexity of solving tropical linear systems
}

\author{
Dima Grigoriev \\ CNRS, Mathématiques, Université de Lille \\ Villeneuve d'Ascq, 59655, France \\ Dmitry.Grigoryev@math.univ-lille1.fr \\ http://en.wikipedia.org/wiki/Dima_Grigoriev
}

\begin{abstract}
An algorithm is designed solving a tropical linear system with the complexity polynomial in the size of the system.
\end{abstract}

\section{Introduction}

A tropical or min-plus semiring (see e. g. [4]) has two operations: min, +. Examples are provided by integers $\mathbb{Z}$, nonnegative integers $\mathbb{Z}^{(\geq 0)}$, real numbers $\mathbb{R}$ or nonnegative real numbers $\mathbb{R}^{(\geq 0)}$. Also the extended semirings $\mathbb{Z}_{\infty}:=\mathbb{Z} \cup\{\infty\}$ and respectively, $\mathbb{Z}_{\infty}^{(\geq 0)}, \mathbb{R}_{\infty}, \mathbb{R}_{\infty}^{(\geq 0)}$ are considered. Studying algorithms we deal mainly with $\mathbb{Z}$ or $\mathbb{Z}_{\infty}$.

We say that a tropical linear system

$$
\min _{1 \leq j \leq n}\left\{a_{i, j}+x_{j}\right\}, 1 \leq i \leq m
$$

(or $(m \times n)$-matrix $\left.A=\left(a_{i, j}\right)\right)$ has a tropical solution $x=\left(x_{1} \ldots, x_{n}\right)$ if for every row $1 \leq i \leq m$ there are two columns $1 \leq k<l \leq n$ such that

$$
a_{i, k}+x_{k}=a_{i, l}+x_{l}=\min _{1 \leq j \leq n}\left\{a_{i, j}+x_{j}\right\}
$$

(see e. g. [3], [12]). Our purpose is to design an algorithm to solve (1).

In Section 1 we assume that coefficients $a_{i, j} \in \mathbb{Z}$ (we call it the case of finite coefficients) and that $0 \leq a_{i, j} \leq M$ for all $i, j$. We describe an algorithm which yields a solution $x \in \mathbb{Z}^{n}$ of (1) or detects its insolvability within the complexity polynomial in $M, n, m$. The algorithm runs by induction on $m$ and starting with any solution of the first $m-1$ equations of (1) the algorithm modifies it in a solution of (1) or detects the insolvability of (1). One can view the algorithm as a tropical analogue of the Gram-Schmidt process with respect to the tropical norm introduced in Section 1.

In Section 2 we study the case of the (extended) coefficients $a_{i, j} \in \mathbb{Z}_{\infty}$ and look for a solution $x \in \mathbb{Z}_{\infty}^{n}$ of (1) with not all its coordinates $x_{j}, 1 \leq j \leq n$ equal $\infty$. We assume 
that $0 \leq a_{i, j} \leq M$ for all finite coefficients. We describe an algorithm which solves (1) also within the complexity polynomial in $M, n, m$. Reordering the columns and rows of $(m \times n)$-matrix $A:=\left(a_{i, j}\right)$ the algorithm brings it to a block form $\left(A_{p, q}\right), 1 \leq p, q \leq t$ such that each of the first $t-1$ diagonal blocks $A_{i, i}, 1 \leq i \leq t-1$ has no (tropical) solution, and all upper-triangular blocks $A_{p, q}, 1 \leq p<q \leq t$ have all entries equal $\infty$. It would be interesting to solve (1) within the complexity polynomial in $\log M, n, m$. However, it is unclear whether the algorithm itself described in Section 2 runs within the latter complexity?

In Section 3 we study tropical non-homogeneous linear systems

$$
\min _{1 \leq j \leq n}\left\{a_{i, j}+x_{j}, a_{i}\right\}, 1 \leq i \leq m
$$

and describe an algorithm for their solving relying on the algorithm from Section 2 with a similar complexity bound.

In Section 4 as a consequence of the algorithm from Section 2 we give a characterization of solvability of (1) in terms of the tropical and Kapranov ranks of matrix $A$ (their definitions are reminded in Section 4) and generalize this characterization to the extended real coefficients from $\mathbb{R}_{\infty}$. Note that for finite coefficients from $\mathbb{R}$ this follows from [7], while for $\mathbb{R}_{\infty}$ the solvability in terms of the tropical rank was established in [9].

In Section 5 we describe an algorithm which tests whether (1) has a unique (in the tropical projective space) solution also within the complexity polynomial in $M, n, m$ (answering a question posed to the author by Thorsten Theobald). It would be interesting to calculate the dimension of the set of tropical solutions of (1) within a similar complexity. The latter problem is apparently difficult since in [3] an example of a linear polynomial ideal is exhibited with an exponential lower bound on the size of its tropical bases.

Note that in [5] it was shown that one can test the tropical singularity of a square matrix in polynomial time. It is known that calculations of the tropical rank [10] and of the Kapranov rank [11] are both NP-hard in general. Moreover, it is proved in [11] that the problem of solving systems of polynomial equations over a given infinite field is reducible to the problem of testing whether the Kapranov rank of a matrix over this field equals 3 . In [12] it was established that solving tropical polynomial systems (already of degrees 2) is NP-complete.

We mention that even in the classical algebra there are known two different notions of a rank of an $(m \times n)$-matrix $A$ over a commutative integral domain $K$ [8]. Define $R k(A)$ to be the minimal $r$ such that $A=X_{1} \cdot Y_{1}+\cdots+X_{r} \cdot Y_{r}$ for suitable $(m \times 1)$-matrices $X_{1}, \ldots, X_{r}$ and $(1 \times n)$-matrices $Y_{1}, \ldots, Y_{r}$ over $K$. Obviously $R k(A)$ is greater or equal to the usual rank $r k(A)$ and can be greater than the latter up to a factor 2 over polynomial rings $K$. 


\section{Solving tropical linear systems with finite coeffi- cients}

In this section we study the case of finite coefficients $a_{i, j} \in \mathbb{Z}$ of system (1) and assume that $0 \leq a_{i, j} \leq M, 1 \leq i \leq m, 1 \leq j \leq n$. Then w.l.o.g. one can look for a solution $x=\left(x_{1}, \ldots, x_{n}\right)$ with coefficients $x_{j} \geq 0,1 \leq j \leq n$ being also integers.

We introduce the notation of the tropical norm of a vector $\|x\|=\sum_{1 \leq j \leq n} x_{j}-n$. $\min _{1 \leq j \leq n}\left\{x_{j}\right\}$. Observe that for the coordinatewise operations min, + on vectors $x, y$ we have $\|\min \{x, y\}\| \leq \max \{\|x\|,\|y\|\}$ and $\|x+y\| \leq\|x\|+\|y\|$. Vector $x$ is equivalent in the tropical projective space [7] to a normalized vector $x-\min _{1 \leq j \leq n}\left\{x_{j}\right\} \cdot(1, \ldots, 1)$. For normalized vectors an inequality $\|\min \{x, y\}\| \leq \min \{\|x\|,\|y\|\}$ holds.

Theorem 1.1 There is an algorithm which for an input (1) either finds its solution or detects its insolvability within complexity $O\left(M \cdot \log M \cdot n^{2} \cdot m^{2}\right)$.

Lemma 1.2 If (1) has a solution $\left(x_{1}, \ldots, x_{n}\right)$ then (1) has a solution $\left(x_{1}^{\prime}, \ldots, x_{n}^{\prime}\right)$ satisfying $0 \leq x_{j}^{\prime} \leq M, x_{j}^{\prime} \leq x_{j}, 1 \leq j \leq n$.

Proof of Lemma 1.2. One can suppose w.l.o.g. that $\min _{1 \leq j \leq n}\left\{x_{j}\right\}=0$. Therefore for each row $i$ it is fulfilled $\min _{1 \leq j \leq n}\left\{a_{i, j}+x_{j}\right\} \leq M$. Hence if column $j_{0}$ satisfies the property $a_{i, j_{0}}+x_{j_{0}}=\min _{1 \leq j \leq n}\left\{a_{i, j}+x_{j}\right\}$ for suitable row $i$ (we call such column $j_{0}$ active) then $x_{j_{0}}^{\prime}:=x_{j_{0}} \leq M$. For any non-active $j_{0}$ one can put $x_{j_{0}}^{\prime}:=\min \left\{x_{j_{0}}, M\right\}$.

Proof of Theorem 1.1 we carry out by induction on $m$. Assume by inductive hypothesis that the algorithm has already produced a current solution $x$ for $((m-1) \times n)$-submatrix $A^{\prime}$ of matrix $A$ excluding the first row of $A$ such that $0 \leq x_{j} \leq M, 1 \leq j \leq n$. Reordering the columns we suppose that $a_{1,1}+x_{1}=\min _{1 \leq j \leq n}\left\{a_{1, j}+x_{j}\right\}$. The algorithm modifies vector $x$ (keeping the property of being a solution of $A^{\prime}$ ) until the modified vector becomes a solution also for the first row or detects that $A$ has no solutions. One can assume that $a_{1,1}+x_{1}<a_{1, j}+x_{j}, j \geq 2$, otherwise the algorithm terminates the inductive step.

We construct by recursion a subset $J$ of columns. At the beginning $J=\{1\}$. For a current $J=\{1, \ldots, k\}$ for each $1 \leq i \leq k$ we have

$$
a_{i, i}+x_{i}=\min _{1 \leq j \leq n}\left\{a_{i, j}+x_{j}\right\}<a_{i, j_{1}}+x_{j_{1}}, j_{1}>i
$$

Suppose that there exists a row $i=i_{k+1}$ for which there is a unique $j_{0} \notin J$ such that $a_{i, j_{0}}+x_{j_{0}}=\min _{1 \leq j \leq n}\left\{a_{i, j}+x_{j}\right\}$. Clearly, $i>k$ due to (3). Transpose column $j_{0}$ with $k+1$ and row $i$ with $k+1$, respectively. Put current $J:=\{1, \ldots, k+1\}$. Then (3) is fulfilled for new $J$.

Now assume that the algorithm fails to augment $J$. Observe that $J$ does not depend on the order of choosing rows $i=i_{k+1}$ in the above construction.

First suppose that $J=\{1, \ldots, n\}$. In this case $(n \times n)$-submatrix of $A$ induced by its first $n$ rows is tropically nonsingular, consequently (1) has no solution and the algorithm halts. 
Now let $k=|J|<n$. If $k=1$ we add to $x_{1}$ number $\min _{2 \leq j \leq n}\left\{a_{1, j}+x_{j}\right\}-\left(a_{1,1}+x_{1}\right) \geq 1$ and obtain a solution of (1). Thereupon apply Lemma 1.2 to the obtained solution, thereby the algorithm terminates the inductive step.

Thus, from now on we assume that $k>1$. We call row $i$ attracted if for every $j_{0}$ such that $a_{i, j_{0}}+x_{j_{0}}=\min _{1 \leq j \leq n}\left\{a_{i, j}+x_{j}\right\}$, we have $j_{0} \in J$. Obviously, the first row is attracted. Reordering the rows one may suppose that exactly first $l$ rows are attracted. Note that for any row $i>l$ there are at least two different columns $j_{1}, j_{2} \notin J$ such that $a_{i, j_{1}}+x_{j_{1}}=a_{i, j_{2}}+x_{j_{2}}=\min _{1 \leq j \leq n}\left\{a_{i, j}+x_{j}\right\}$.

For $1 \leq i \leq l$ denote

$$
a_{i}:=\min _{k<j \leq n}\left\{a_{i, j}+x_{j}\right\}-\min _{1 \leq j \leq n}\left\{a_{i, j}+x_{j}\right\} \geq 1
$$

and $a:=\min _{1 \leq i \leq l}\left\{a_{i}\right\}$. The algorithm modifies vector $\left(x_{1}, \ldots, x_{n}\right)$ in such a way that

$$
y_{j}:=x_{j}+a, 1 \leq j \leq k ; \quad y_{j}:=x_{j}, j>k .
$$

Then vector $y:=\left(y_{1}, \ldots, y_{n}\right)$ is still a solution of $A^{\prime}$ and $a_{1,1}+y_{1}=\min _{1 \leq j \leq n}\left\{a_{1, j}+y_{j}\right\}$. Moreover, the tropical norm

$$
\left\|\left(a_{1, j}+y_{1}, \ldots, a_{1, n}+y_{n}\right)\right\|=\left\|\left(a_{1, j}+x_{1}, \ldots, a_{1, n}+x_{n}\right)\right\|-a \cdot(n-k)
$$

has dropped.

Thereupon the algorithm applies Lemma 1.2 to vector $\left(y_{1}, \ldots, y_{n}\right)$. Observe that this does not change the tropical norm since each of the first $k$ columns is active (taking into account that $k>1$ ) and hence every $y_{j} \leq M, j>k$ and thereby $y_{j}$ does not change in the course of application of Lemma 1.2.

Thus, we have described a single iteration of the algorithm. The nest iteration starts with the modified vector $y$ replacing $x$. The complexity of the execution of the iteration can be bounded by $O(\log M \cdot m \cdot n)$. The total number of iterations does not exceed tropical norm $\left\|\left(a_{1, j}+x_{1}, \ldots, a_{1, n}+x_{n}\right)\right\| \leq 2 \cdot M \cdot(n-1)$. Since the described induction (considering each time one more row of matrix $A$ ) requires $m$ steps, we conclude the complexity bound in Theorem 1.1.

\section{Solving tropical linear systems with coefficients ex- tended by infinity}

From now on we assume that entries of (1) $a_{i, j} \in \mathbb{Z}_{\infty}$ and $0 \leq a_{i, j} \leq M$ when $a_{i, j} \in \mathbb{Z}$. We are looking for solutions $\left(x_{1}, \ldots, x_{n}\right)$ over $\mathbb{Z}_{\infty}$ with not all the coordinates equal $\infty$.

Theorem 2.1 There is an algorithm which for a tropical linear system (1) over $\mathbb{Z}_{\infty}$ either finds a solution or detects its insolvability within complexity $O\left(M \cdot \log (M \cdot n) \cdot n^{4} \cdot m^{2}\right)$. 
Lemma 2.2 If $\left(x_{1}, \ldots, x_{n}\right) \in\left(\mathbb{R}_{\infty}\right)^{n}$ is a solution of (1) then there exists a solution $\left(x_{1}^{\prime}, \ldots, x_{n}^{\prime}\right)$ of (1) such that for any $1 \leq j, j_{1} \leq n$ it holds:

- $x_{j}^{\prime}=\infty$ iff $x_{j}=\infty$;

- $0 \leq x_{j}^{\prime} \leq \min \left\{x_{j},(M+1) \cdot n\right\}$, provided that $x_{j} \neq \infty$;

- $x_{j}-x_{j_{1}}>M$ iff $x_{j}^{\prime}-x_{j_{1}}^{\prime}>M$.

Proof of Lemma 2.2. In the course of the proof we will modify vector $\left(x_{1}, \ldots, x_{n}\right)$ keeping for it the same notation. One can assume w.l.o.g. that $0=x_{1}=\min _{1 \leq j \leq n}\left\{x_{j}\right\}$. Consider a graph whose vertices are finite coordinates $x_{j}$, and a pair of coordinates $x_{p}, x_{q}$ is connected by an edge if for some row $i$ we have $a_{i, p}+x_{p}=a_{i, q}+x_{q}=\min _{1 \leq j \leq n}\left\{a_{i, j}+x_{j}\right\} \neq$ $\infty$.

Consider a connected component of the graph which contains $x_{1}$. Let the component contain $p$ vertices, and after their reordering one can assume that it consists of $x_{1}, \ldots, x_{p}$, hence $x_{j} \leq M \cdot(p-1), 1 \leq j \leq p$. After reordering the coordinates one can assume that $x_{p+1}=\min _{j>p}\left\{x_{j}\right\}$. If $x_{p+1}=\infty$ the Lemma is proved. Otherwise, if $x_{p+1} \geq M \cdot p+1$ then replace $x_{j}$ with $x_{j}-x_{p+1}+(M \cdot p+1)$ for all $j>p$. Take a connected component of the graph which contains $x_{p+1}$. Let it consist of $q$ vertices $x_{p+1}, \ldots, x_{p+q}$. As above we conclude that $x_{p+j} \leq M \cdot(p+q-1)+1,1 \leq j \leq q$. Continuing in this way we complete the proof of Lemma.

The proof of Theorem 2.1 we carry out by induction on $m$ and first formulate the inductive hypothesis. Suppose that $((m-1) \times n)$-submatrix $A^{\prime}$ of $A$ (after reordering the rows and columns) has a block structure

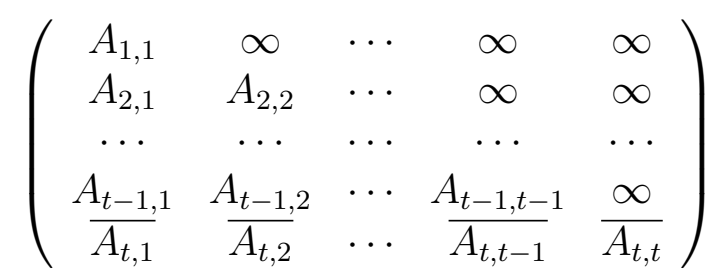

where $A_{p, q}$ is of size $u_{p} \times v_{q}, 1 \leq p, q \leq t-1$, while the lowest blocks $\overline{A_{t, q}}$ are of sizes $\overline{u_{t}} \times v_{q}, 1 \leq q<t$, the rightmost blocks $\overline{A_{p, t}}$ are of sizes $u_{p} \times \overline{v_{t}}, 1 \leq p<t$, finally the diagonal block $\overline{A_{t, t}}$ is of size $\overline{u_{t}} \times \overline{v_{t}}$ where $\overline{u_{t}}=m-1-u_{1}-\cdots-u_{t-1}, \overline{v_{t}}=n-v_{1}-\cdots-v_{t-1}$.

Also a vector $\left(y_{1}, \ldots, y_{n}\right) \in \mathbb{Z}^{n}, 0 \leq y_{j} \leq(M+1) \cdot n, 1 \leq j \leq n$ is yielded. For each diagonal block $A_{p, p}=\left(a_{u+i, v+j}\right), 1 \leq p<t, u:=u_{1}+\cdots+u_{p-1}, 1 \leq i \leq u_{p}, v:=$ $v_{1}+\cdots+v_{p-1}, 1 \leq j \leq v_{p}$ (except for the lowest diagonal block $\overline{A_{t, t}}$ ) we have $a_{u+i, v+i}+y_{v+i}=$ $\min _{1 \leq j \leq v_{p}}\left\{a_{u+i, v+j}+y_{v+j}\right\}$ for $1 \leq i \leq v_{p}$ and $a_{u+i, v+i}+y_{v+i}<a_{u+i, v+j}+y_{v+j}, i<j \leq v_{p}$. Therefore, in particular $u_{p} \geq v_{p}, p<t$. It is not excluded that $\overline{u_{t}}=0$, while the case $\overline{v_{t}}=0$ would mean that the algorithm under description terminates with the output that system (1) has no solutions (cf. below Lemma 2.5).

Every entry of each upper-triangular block $A_{p, q}, p<q$ (as well as of $\overline{A_{p, t}}, p<t$ ) equals $\infty$. Moreover, the vector from $\mathbb{Z}_{\infty}^{n}$ whose coordinates in the first $t-1$ blocks equal $\infty$ and in the last $t$-th block coincide with $y_{j}$ for $v_{1}+\cdots+v_{t-1}<j \leq n$, is a tropical solution of matrix $A^{\prime}$. 
For the sake of simplifying notations denote matrix $B^{\prime}=\overline{A_{t, t}}$ of size $\left(r-1:=\overline{u_{t}}\right) \times(s:=$ $\left.\overline{v_{t}}\right)$. One can assume that $B^{\prime}$ has no rows consisting fully of $\infty$ entries, otherwise the corresponding row of matrix $A^{\prime}$ one can join to the previous $(t-1)$-th block.

We assume that matrix $A^{\prime}$ is obtained from $A$ by deleting its $(m-r+1)$-th row. By $B=\left(b_{i, j}\right), 1 \leq i \leq r, 1 \leq j \leq s$ denote $(r \times s)$-submatrix of $A$ located in its lower right corner. Deleting the first row from $B$ we obtain $B^{\prime}$. Also one can suppose w.l.o.g. that $b_{1,1}+y_{n-s+1}=\min _{1 \leq j \leq s}\left\{b_{1, j}+y_{n-s+j}\right\}$.

The algorithm will modify vector $\left(y_{n-s+1}, \ldots, y_{n}\right)$ (keeping for a current vector the same notation) while preserving the property that $\left(y_{n-s+1}, \ldots, y_{n}\right)$ is a (tropical) solution of $B^{\prime}$. One can assume w.l.o.g. that $b_{1,1}+y_{n-s+1}<\min _{2 \leq j \leq s}\left\{b_{1, j}+y_{n-s+j}\right\}$, since otherwise the vector from $\mathbb{Z}_{\infty}^{n}$ with all coordinates in the first $t-1$ blocks equal $\infty$ and coinciding with vector $\left(y_{n-s+1}, \ldots, y_{n}\right)$ in the last $t$-th block provides a solution of $A$ which would terminate the inductive step. In this case in the block structure $u_{1}, \ldots, u_{t-1}, v_{1}, \ldots, v_{t-1}, \overline{v_{t}}$ do not change, while $\overline{u_{t}}$ increases by one. Applying Lemma 2.2 one can assume w.l.o.g. that $0 \leq y_{j} \leq(M+1) \cdot n, n-s+1 \leq j \leq n$.

As above in the proof of Theorem 1.1 the algorithm constructs recursively a set $J \subset$ $\{n-s+1, \ldots, n\}$ of columns of matrix $B$, while modifying vector $\left(y_{n-s+1}, \ldots, y_{n}\right)$, and we describe a single iteration of this modification. As in the proof of Theorem $1.1(n-s+1) \in$ $J$. Again as above introduce the set of attracted rows. For every attracted row $i$ denote

$$
b_{i}:=\min _{j \notin J}\left\{b_{i, j-n+s}+y_{j}\right\}-\min _{j \in J}\left\{b_{i, j-n+s}+y_{j}\right\} \geq 1 .
$$

Then $b:=\min \left\{b_{i}\right\} \geq 1$ where $\min$ is taken over all the attracted rows. Thus, the algorithm modifies vector $\left(y_{n-s+1}, \ldots, y_{n}\right)$ adding $b$ to every $y_{j}$ for $j \in J$. Thereupon the algorithm applies Lemma 2.2 to vector $\left(y_{n-s+1}, \ldots, y_{n}\right)$ which satisfies $B^{\prime}$. Hence one can assume w.l.o.g. that $0 \leq y_{j} \leq(M+1) \cdot s, n-s+1 \leq j \leq n$.

The algorithm introduces the following directed graph $G$ with $s$ vertices $\{n-s+$ $1, \ldots, n\}$. There is an edge in $G$ from $j_{1}$ to $j_{2}$ if $y_{j_{1}}-y_{j_{2}} \leq M$. Observe that an application of Lemma 2.2 to vector $\left(y_{n-s+1}, \ldots, y_{n}\right)$ does not change graph $G$. Denote by $S \subset\{n-s+1, \ldots, n\}$ the set of all the vertices which can be reached in $G$ starting with vertex $n-s+1$. In the course of executing the algorithm, while modifying $J, G, S$ we keep for them the same notations.

Lemma 2.3 After any subsequent iteration of the algorithm set $J$ remains to be a subset of $S$. Set $S$ after an iteration becomes a subset of $S$ before the iteration.

Proof of Lemma 2.3. At the current iteration inclusion $J \subset S$ holds by virtue of construction of $J$ since for any row $i$ and any pair of columns $j \in S, l \notin S$ we have $b_{i, l-n+s}+y_{l} \neq b_{i, j-n+s}+y_{j}$, unless $b_{i, l-n+s}=b_{i, j-n+s}=\infty$. Therefore, after the modification of vector $\left(y_{n-s+1}, \ldots, y_{n}\right)$ its coordinates $y_{j}$ for $j \in J$ increase, while the other coordinates do not change. Consequently, the modified $S$ is a subset of the previous $S$.

Lemma 2.4 For any attracted row $i$ and any $l \notin S$ we have $b_{i, l-n+s}=\infty$. 
Proof of Lemma 2.4. If $b_{i, l-n+s}<\infty$ then $b_{i, l-n+s}+y_{l}<b_{i, j-n+s}+y_{j}$ for any $j \in S$ which contradicts to that row $i$ is attracted and that $J \subset S$ (due to Lemma 2.3).

Now assume that $J=S$. Denote by $v_{t}:=\# J$ and by $u_{t}$ the number of attracted rows of $B$. Reorder the rows and the columns of $B$ (and respectively, of $A$ ) in such a way that the set of the first $v_{t}$ columns of $B$ coincides with $J$, and the set of the first $u_{t}$ rows of $B$ coincides with the set of attracted rows of $B$. Moreover, one can suppose that for any $1 \leq i \leq v_{t}$ we have

$$
b_{i, i}+y_{n-s+i}=\min _{1 \leq j \leq s}\left\{b_{i, j}+y_{n-s+j}\right\}<\min _{i<l \leq s}\left\{b_{i, l}+y_{n-s+l}\right\} .
$$

Then the algorithm constructs a modified block decomposition of $A$ being a refinement of the block decomposition from the inductive hypothesis: the last $\overline{u_{t}}$ rows (respectively, the last $\overline{v_{t}}$ columns) of $A$ are partitioned into the first $u_{t}$ rows and the remaining $\overline{u_{t+1}}:=$ $\overline{u_{t}}-u_{t}$ rows (respectively, into the first $v_{t}$ columns and the remaining $\overline{v_{t+1}}:=\overline{v_{t}}-v_{t}$ columns). Thus, as blocks of $A$ we obtain the new ones $A_{t, q}, q \leq t ; A_{p, t}, p \leq t ; \overline{A_{t+1, q}}, q \leq$ $t+1 ; \overline{A_{p, t+1}}, p \leq t+1$. The diagonal block $A_{t, t}$ satisfies the inductive hypothesis by its construction, see (4), and each entry of $\overline{A_{t, t+1}}$ equals $\infty$ due to Lemma 2.4. This completes the inductive step for $m$ rows (i. e. for matrix $A$ ).

The algorithm terminates when it is impossible to continue its work. It can happen when either all the rows of (1) or all its columns are exhausted. First consider the case when all the rows of (1) are exhausted (i. e. A contains all the rows of (1)), but not all the columns are exhausted. Then two possibilities can occur. Either a (modified) vector $\left(y_{n-s+1}, \ldots, y_{n}\right)$ is a solution of matrix $B$, then the algorithm terminates before completing a block decomposition of matrix $A$ (at the inductive step) and outputs a solution of (1) (see above). Or the inductive step is completed with all the rows of $B$ being attracted (since all the rows of (1) are exhausted) and with $J=S \neq\{n-s+1, \ldots, n\}$ (since not all the columns of (1) are exhausted). In the latter case $\overline{u_{t}}=u_{t}$, in other words blocks $\overline{A_{t+1, q}}$ are void, block $\overline{A_{t, t+1}}$ is not empty with each its entry equal $\infty$. Then (1) has a solution whose coordinates at first $t$ blocks equal $\infty$ and at $(t+1)$-th block equal, say, 0 (or other arbitrary integers).

Secondly, consider the case when all the columns of (1) are exhausted, i. e. $J=\{n-$ $s+1, \ldots, n\}$. Then observe that $\overline{u_{t}}=u_{t}, \overline{v_{t}}=v_{t}$, thus blocks $\overline{A_{t+1, q}} ; \overline{A_{p, t+1}}, 1 \leq p, q \leq t+1$ being void. Consider $(n \times n)$-submatrix $\tilde{C}=\left(\tilde{c}_{i, j}\right)$ of $A$ consisting of its first $v_{p}$ rows from each block of decomposition of $A, 1 \leq p \leq t$. Denote by $C=\left(c_{i, j}\right)$ matrix such that $c_{i, j}:=\tilde{c}_{i, j}+y_{j}, 1 \leq i, j \leq n$. Evidently, the tropical linear systems with matrices $\tilde{C}$ and $C$ have solutions simultaneously.

Lemma 2.5 Let $(n \times n)$-matrix $C$ be decomposed into blocks $C_{p, q}$ of sizes $v_{p} \times v_{q}, 1 \leq$ $p, q \leq t ; n=v_{1}+\cdots+v_{t}$. Moreover, for each diagonal block $C_{p, p}=\left(c_{\bar{v}+i, \bar{v}+j}\right), 1 \leq i, j \leq$ $v_{p}, 1 \leq p \leq t$ where $\bar{v}=v_{1}+\cdots v_{p-1}$ we have

$$
c_{\bar{v}+i, \bar{v}+i}=\min _{1 \leq j \leq v_{p}}\left\{c_{\bar{v}+i, \bar{v}+j}\right\}<\min _{i<l \leq v_{p}}\left\{c_{\bar{v}+i, \bar{v}+l}\right\}
$$


for every $1 \leq i \leq v_{p}$. In addition, any entry of an upper-triangular block $C_{p, q}, p<q$ equals $\infty$. Then a tropical linear system with matrix $C$ has no solution over $\mathbb{Z}_{\infty}$.

Proof of Lemma 2.5. Suppose that vector $\left(z_{1}, \ldots, z_{n}\right)$ is a tropical solution of matrix $C$. Let $p$ be the first block $\left(z_{\bar{v}+1}, \ldots, z_{\bar{v}+v_{p}}\right)$ of $\left(z_{1}, \ldots, z_{n}\right)$ which contains a finite coordinate. Then $\left(z_{\bar{v}+1}, \ldots, z_{\bar{v}+v_{p}}\right)$ is a tropical solution of matrix $C_{p, p}$. Take a unique $1 \leq j_{0} \leq v_{p}$ such that

$$
z_{\bar{v}+j_{0}}=\min _{1 \leq j \leq v_{p}}\left\{z_{\bar{v}+j}\right\}<\min _{1 \leq j \leq j_{0}}\left\{z_{\bar{v}+j}\right\} .
$$

Then we conclude that $\left(z_{\bar{v}+1}, \ldots, z_{\bar{v}+v_{p}}\right)$ is not a tropical solution of $j_{0}$-th row of matrix $C_{p, p}$ because $c_{\bar{v}+j_{0}, \bar{v}+j_{0}}+z_{\bar{v}+j_{0}}<\min _{1 \leq j \leq v_{p}, j \neq j_{0}}\left\{c_{\bar{v}+j_{0}, \bar{v}+j}+z_{\bar{v}+j}\right\}$. The achieved contradiction proves the Lemma.

Lemma 2.5 implies the correctness of the described algorithm: it outputs a solution of (1) over $\mathbb{Z}_{\infty}$ iff (1) is solvable.

Now we estimate the complexity of the algorithm. We recall that in the course of an iteration modifying vector $\left(y_{n-s+1}, \ldots, y_{n}\right)$ the modified set $S$ becomes a subset of the previous set $S$ (see Lemma 2.3). First we bound from above the number of iterations while $S$ does not change. Observe that the integer $N:=(s-1) \cdot y_{n-s+1}-y_{n-s+2}-\cdots-y_{n}$ increases after every iteration because the algorithm adds an integer $b \geq 1$ to each $y_{j}$ for $j \in J \subset S$ (due to Lemma 2.3), while $n-s+1 \in J$, in addition $J \neq S$ (otherwise, the algorithm completes the inductive step). At the beginning of the inductive step $N \geq$ $-(s-1) \cdot(M+1) \cdot n$ (cf. Lemma 2.2). If $N$ becomes larger than $M \cdot s^{2}$ then $S$ should change (since not all the vertices of $S$ become reachable in graph $G$ ). Therefore, after at most of $O(M \cdot s \cdot n)$ iterations set $S$ changes. Again due to Lemma $2.3 S$ can be modified at most $s$ times. Thus, the whole number of iterations in the inductive step is less than $O\left(M \cdot s^{2} \cdot n\right) \leq O\left(M \cdot n^{3}\right)$.

The complexity of executing a single iteration is bounded by $\log (M \cdot n) \cdot m \cdot n$ (cf. Lemma 2.2). The number of inductive steps (augmenting the set of rows of (1) under consideration) does not exceed $m$. Summarizing, this provides the complexity bound $O(M$. $\left.\log (M \cdot n) \cdot n^{4} \cdot m^{2}\right)$ of the algorithm and completes the proof of Theorem 2.1.

When the paper was already submitted the author has learned that a different algorithm for solving tropical linear systems was designed in [1] with a similar complexity bound as in Theorem 2.1 (implying also Corollary 4.2 below). The approach from [1] involves mean payoff games and provides in addition an algorithm for solving min-linear systems [4]

$$
\min _{1 \leq j \leq n_{1}}\left\{a_{i, j}+x_{j}\right\}=\min _{1 \leq l \leq n_{2}}\left\{b_{i, l}+y_{l}\right\}, 1 \leq i \leq m
$$

For the first time an algorithm for solving system (5) within the similar complexity bound polynomial in $n_{1}, n_{2}, m, M$ was proposed in [6]. In [2] there was produced an example of system (5) with sizes $n_{1}=n_{2}=2, m=3$ and $a_{1,1}=a_{1,2}=b_{1,2}=1, a_{2,2}=$ $b_{2,2}=M$ (the remaining entries vanish) for which the algorithm from [6] runs with the complexity lower bound polynomial in $M$. 
Observe that an example of this sort (with matrices of a constant size) for the algorithm from Theorem 2.1 (for a different problem of solving a tropical linear system (1)) would be impossible, because the algorithm from Theorem 2.1 runs actually within the complexity polynomial in $\exp (n \cdot m), \log M$. Indeed, for each $t$ and row $1 \leq i \leq r$ of matrix $B$ consider the set of columns $1 \leq j \leq s$ such that $b_{i, j}+y_{n-s+j}=\min _{1 \leq l \leq s}\left\{b_{i, l}+y_{n-s+l}\right\}$ (cf. (4)). One can verify that the sets of all such pairs $i, j$ are distinct at different steps of the algorithm. On the other hand, it is unclear whether the algorithm from Theorem 2.1 runs within the complexity polynomial in $n, m, \log M$ (rather than in $M)$ ?

\section{Solving tropical non-homogeneous linear systems}

Treating (1) as a tropical homogeneous linear system one can consider its non-homogeneous counterpart (2). Denote by $\hat{A}$ the matrix of size $m \times(n+1)$ obtained from $A=\left(a_{i, j}\right)$ by joining as the last $(n+1)$-th column $\left(a_{1}, \ldots, a_{m}\right)^{T}$. Then $(2)$ has a tropical solution over $\mathbb{Z}_{\infty}$ iff the homogeneous linear system with the matrix $\hat{A}$ has a tropical solution $\left(x_{1}, \ldots, x_{n}, x_{n+1}\right)$ such that $x_{n+1} \neq \infty$. We describe an algorithm which can test the existence of such a solution.

The algorithm from Theorem 2.1 brings matrix $\hat{A}$ (after handling all its $m$ rows) to the block form $\left(A_{p, q}\right)$ with block sizes $u_{1}, \ldots, u_{t} ; v_{1}, \ldots, v_{t}$ (possibly $u_{t}=0$ ). We assume that the homogeneous system with the matrix $\hat{A}$ has a tropical solution (which is detected by the algorithm from Theorem 2.1), otherwise (2) has no tropical solution.

The proof of Lemma 2.5 entails that any solution of the homogeneous system with the matrix $\hat{A}$ has coordinates equal $\infty$ in the first $t-1$ blocks of sizes $v_{1}, \ldots, v_{t-1}$ (we remind that the algorithm from Theorem 2.1 reorders the columns and rows of $\hat{A}$ ). On the other hand, there is a solution with all finite coordinates in the last $t$-th block of size $v_{t}$. Thus, the criterion of solvability of (2) is that the last $(n+1)$-th column of $\hat{A}$ belongs to the last $t$-th block.

Assume that the entries $a_{i, j}, a_{i}$ satisfy the same bounds as $a_{i, j}$ from (1). Making use of Theorem 2.1, we get

Corollary 3.1 There is an algorithm which for an input (2) either finds its solution over $\mathbb{Z}_{\infty}$ or detects its insolvability within complexity $O\left(M \cdot \log (M \cdot n) \cdot n^{4} \cdot m^{2}\right)$.

\section{Solvability of tropical linear systems via tropical and Kapranov ranks}

As a direct consequence of Theorem 2.1 we get a criterion of solvability of a tropical linear system (1) over $\mathbb{Z}_{\infty}$ in terms of its tropical and Kapranov ranks [7].

Similar to matrices over $\mathbb{Z}$ we call $(n \times n)$-matrix $A=\left(a_{i, j}\right)$ tropically non-singular if there exists a unique assignment $\left\{a_{i, \pi(i)}\right\}_{1 \leq i \leq n}$ for a permutation $\pi \in \operatorname{Sym}(n)$ with a minimal sum $\sum_{1 \leq i \leq n} a_{i, \pi(i)}$ (in this case the latter sum is obviously finite). Then as 
usually, the tropical rank of an $(m \times n)$-matrix is defined as the maximal size of tropically non-singular submatrices.

For an $(m \times n)$-matrix $A=\left(a_{i, j}\right)$ its lifting is defined as an $(m \times n)$-matrix $F=\left(f_{i, j}\right)$ over the field of Puiseux series $K=\mathbb{C}\left(\left(t^{1 / \infty}\right)\right)$ such that $\operatorname{ord}\left(f_{i, j}\right)=a_{i, j}$ or $f_{i, j}=0$ when $a_{i, j}=\infty$. Then the Kapranov rank of $A$ is said to be less or equal to $r$ if there exists a lifting $F$ of $A$ with the rank (over $K$ ) at most $r$.

Corollary 4.1 The following three statements are equivalent:

i) A tropical linear system (1) with $(m \times n)$-matrix $A$ has a solution over $\mathbb{Z}_{\infty}$;

ii) The tropical rank of $A$ is less than $n$;

iii) The Kapranov rank of $A$ is less than $n$.

Proof of Corollary 4.1. The implication iii) $\Rightarrow$ ii) is evident (cf. e. g. [7]). In [7] it is also shown the equivalence of ii) and iii) for matrices over $\mathbb{R}$ (so, with finite coefficients). Also the equivalence of i) and ii) was established in [9].

The implication ii) $\Rightarrow$ i) follows from Theorem 2.1. Indeed, if (1) has no solutions, the algorithm designed in the proof of Theorem 2.1 terminates by exhausting the columns of (1). Hence there is an $(n \times n)$-submatrix $\tilde{C}=\left(\tilde{c}_{i, j}\right)$ of $A$ such that $(n \times n)$-matrix $C=\left(c_{i, j}\right)$ for which $c_{i, j}=\tilde{c}_{i, j}+y_{j}$ for an appropriate vector $\left(y_{1}, \ldots, y_{n}\right) \in \mathbb{Z}^{n}$ fulfils the properties of Lemma 2.5. Clearly, matrix $C$ has a unique minimal assignment located on its diagonal and thereby is tropically non-singular, the same holds for $\tilde{C}$ as well.

To establish the remaining implication i) $\Rightarrow$ iii) consider a solution $\left(x_{1}, \ldots, x_{n}\right) \in\left(\mathbb{Z}_{\infty}\right)^{n}$ of $A$. We take a vector $z:=\left(z_{1}, \ldots, z_{n}\right) \in K^{n}$ such that $z_{j}=t^{x_{j}}$ or $z_{j}=0$ when $x_{j}=\infty$. Our purpose is to produce an $(m \times n)$-matrix $F=\left(f_{i, j}\right)$ over $K$ such that $F \cdot z=0$ and $\operatorname{ord}\left(f_{i, j}\right)=a_{i, j}$ or $f_{i, j}=0$ when $a_{i, j}=\infty$ (i. e. $F$ will be a lifting of $A$ ).

Fix a row $i$ for the time being. If $\min _{1 \leq j \leq n}\left\{a_{i, j}+x_{j}\right\}=\infty$ we have $f_{i, j} \cdot z_{j}=0,1 \leq$ $j \leq n$. Now let $a_{i, l}+x_{l}=\min _{1 \leq j \leq n}\left\{a_{i, j}+x_{j}\right\}<\infty$ for all $l \in L$ for a certain subset $L \subset\{1, \ldots, n\}$ with at least two elements. We look for $f_{i, j}=\sum_{k \geq a_{i, j}} g_{j, k} \cdot t^{k}$ as polynomials with indeterminate coefficients $g_{j, k} \in \mathbb{Z}$. Fix in an arbitrary way all $f_{i, j}:=t^{a_{i, j}}$ (when $\left.a_{i, j}<\infty\right)$ for all $j$ except a single $l_{0} \in L$. Expanding equality $\sum_{1 \leq j \leq n} f_{i, j} \cdot z_{j}=0$ in the powers of $t$ we obtain in the unique way a polynomial $f_{i, l_{0}}=-(\# L-1) \cdot t^{a_{i, l_{0}}}+\cdots \in \mathbb{Z}[t]$ with ord $\left(f_{i, l_{0}}\right)=a_{i, l_{0}}$. Since the rank of $F$ (being a lifting of $A$ ) is less than $n$, we establish iii).

Clearly, one can detect solvability of (1) by verifying the tropical singularity of all $(n \times n)$-submatrices of $A$ (see Corollary 4.1 ), thus within the complexity polynomial in $\log M,\left(\begin{array}{c}m \\ n\end{array}\right)$, cf. [5].

Corollary 4.2 The problem of solvability of a tropical linear system belongs to the complexity class $N P \cap \operatorname{coNP}$.

Remark 4.3 For (extended) rational coefficients $a_{i, j} \in \mathbb{Q}_{\infty}$ Theorem 2.1 and Corollary 4.1 hold literally. 
Remark 4.4 For (extended) real coefficients $a_{i, j} \in \mathbb{R}_{\infty}$ statements i) and ii) of Corollary 4.1 are equivalent. Indeed, for the implication ii) $\Rightarrow$ i) one can in the proof of Theorem 2.1 replace the induction with the transfinite induction, while modifying vector $\left(y_{n-s+1}, \ldots, y_{n}\right)$ and proving existence of a solution of (1) (again matrix C from Lemma 2.5 is tropically non-singular).

To prove the inverse implication i) $\Rightarrow$ ii) assume that $\left(x_{1}, \ldots, x_{n}\right) \in\left(\mathbb{R}_{\infty}\right)^{n}$ is a solution of a tropical square linear system (1), i. e. $m=n$, and that $A$ has a unique minimal assignment. Reordering the rows and the columns of $A$ one can suppose w.l.o.g. that $x_{j}=\infty$ iff $j>k$ and in addition that the unique minimal assignment is located on the diagonal of $A$. Then vector $\left(x_{1}, \ldots, x_{k}\right) \in \mathbb{R}^{k}$ is a solution of $(k \times k)$-submatrix $A_{k}=$ $\left(a_{i, j}\right), 1 \leq i, j \leq k$ of $A$ in its upper left corner. Consider a directed graph $H$ with $k$ vertices $x_{1}, \ldots, x_{k}$. For a pair of vertices $x_{i}, x_{j}, i \neq j$ there is an edge $\left(x_{i}, x_{j}\right)$ in $H$ if $a_{i, j}+x_{j}=\min _{1 \leq l \leq k}\left\{a_{i, l}+x_{l}\right\}$. Since $\left(x_{1}, \ldots, x_{k}\right)$ is a solution of $A_{k}$, for any $1 \leq i \leq k$ there is $1 \leq j \leq k$ such that $H$ contains edge $\left(x_{i}, x_{j}\right)$. Therefore, there exists a simple cycle $x_{i_{1}}, x_{i_{2}}, \ldots, x_{i_{s}}$ in $H$. Then the assignment of $A$ obtained from the diagonal one by means of replacing $a_{i_{1}, i_{1}}, a_{i_{2}, i_{2}}, \ldots, a_{i_{s}, i_{s}}$ with $a_{i_{1}, i_{2}}, a_{i_{2}, i_{3}}, \ldots, a_{i_{s}, i_{1}}$, has the same sum as the diagonal one. This contradiction with the tropical singularity of $A$ proves ii).

\section{Testing uniqueness of a solution of a tropical linear system}

Let $y=\left(y_{1}, \ldots, y_{n}\right) \in \mathbb{Z}_{\infty}^{n}$ be a solution of (1) (being yielded, say, by the algorithm designed in Theorem 2.1). One can suppose w.l.o.g. that $0 \leq y_{j} \leq(M+1) \cdot n+1$ when $y_{j}$ is finite for $1 \leq j \leq n$, cf. Lemma 2.2. Our purpose is to test whether $y$ is unique (in the tropical projective space [7]) solution of (1). We refer to two vectors as different if they are different in the tropical projective space. The set $S_{\infty}(y) \subset\{1, \ldots, n\}$ of all $1 \leq l \leq n$ such that $y_{l}=\infty$ we call the infinity support of $y$.

Lemma 5.1 Assume that there exists a solution $z=\left(z_{1}, \ldots, z_{n}\right) \in \mathbb{Z}_{\infty}^{n}$ of (1) different from $y$. Then there exists a solution $w=\left(w_{1}, \ldots, w_{n}\right) \in \mathbb{Z}_{\infty}^{n}$ of (1) and a pair of indices $1 \leq j \neq l \leq n$ with $\{j, l\} \not \subset S_{\infty}(y)$ and $j, l \notin S_{\infty}(w)$ such that

i) if $j, l \notin S_{\infty}(y)$ then $w_{l} \leq y_{l}, w_{j} \leq y_{j}, y_{l}-w_{l}+y_{j}-w_{j}=1$;

ii) if $j \in S_{\infty}(y), l \notin S_{\infty}(y)$ then $w_{j}-w_{l}=(M+1) \cdot n$.

Moreover, $S_{\infty}(w)=S_{\infty}(y) \cap S_{\infty}(z)$.

Proof of Lemma 5.1. One can suppose w.l.o.g. that $0 \leq z_{j} \leq(M+1) \cdot n, 1 \leq j \leq n$, cf. Lemma 2.2, and still $y$ and $z$ are different. If among three sets $S_{\infty}(y) \backslash S_{\infty}(z), S_{\infty}(z) \backslash$ $S_{\infty}(y),\{1, \ldots, n\} \backslash\left(S_{\infty}(y) \cup S_{\infty}(z)\right)$ at least two are nonempty, pick $j$ from one of them and $l$ from another one. Otherwise, $S_{\infty}(y) \backslash S_{\infty}(z)=S_{\infty}(z) \backslash S_{\infty}(y)=\emptyset$, in this case as $j, l$ pick any two elements from $\{1, \ldots, n\} \backslash\left(S_{\infty}(y) \cup S_{\infty}(z)\right)$ with the property that $y_{l}-z_{l} \neq y_{j}-z_{j}$ (such $j, l$ exist since $y, z$ are different). 
First consider the case when $j, l \notin S_{\infty}(y)$. Vector $z^{\prime}:=z+\left(\max \left\{y_{l}-z_{l}, y_{j}-z_{j}\right\}-\right.$ $1) \cdot(1, \ldots, 1)$ is a solution of $(1)$ (note that $\max \left\{y_{l}-z_{l}, y_{j}-z_{j}\right\} \in \mathbb{Z}$ because not both $z_{j}, z_{l}$ equal $\infty$ by virtue of the choice of $\left.j, l\right)$. Put $w:=\left(w_{1}, \ldots, w_{n}\right):=\min \left\{y, z^{\prime}\right\}$. Let for definiteness $y_{l}-z_{l}>y_{j}-z_{j}$. Then $w_{l}=y_{l}-1, w_{j}=y_{j}$ which proves Lemma in the first case.

Secondly, assume that $j \in S_{\infty}(y), l \notin S_{\infty}(y)$. Vector $y^{\prime}:=y+\left(z_{j}-(M+1) \cdot n-\right.$ $\left.y_{l}\right) \cdot(1, \ldots, 1)$ is a solution of $(1)$, put $w:=\left(w_{1}, \ldots, w_{n}\right):=\min \left\{y^{\prime}, z\right\}$. Then $w_{j}=z_{j}$ and $w_{l}=y_{l}^{\prime}=z_{j}-(M+1) \cdot n \leq z_{l}$ due to the supposition above.

Now we describe an algorithm which tests whether $y$ is a unique solution of (1). For each pair $\{j, l\} \not \subset S_{\infty}(y)$ the algorithm chooses integers $w_{j}, w_{l}$ with $\min \left\{w_{j}, w_{l}\right\}=0$ which satisfy either i) or ii) from Lemma 5.1. The algorithm extends (1) by a tropical linear equation $\min \left\{w_{l}+x_{j}, w_{j}+x_{l}\right\}$ and applies to the extended system Theorem 2.1. Lemma 5.1 implies that if $y$ is not a unique solution of (1) then the extended system for at least one of the pairs $\{j, l\} \not \subset S_{\infty}(y)$ will have a solution. Conversely, if the extended system has a solution $x=\left(x_{1}, \ldots, x_{n}\right)$ then either $x_{j}, x_{l} \in \mathbb{Z}, x_{j}-x_{l}=w_{j}-w_{l}$ or $x_{j}=x_{l}=\infty$. In any of two latter cases vector $x$ differs from $y$. Summarizing and employing Theorem 2.1 we conclude with the following

Corollary 5.2 There is an algorithm which for a given system (1) tests whether it has a unique solution, within the complexity $O\left(M \cdot \log (M \cdot n) \cdot n^{7} \cdot m^{2}\right)$.

It is an open question whether one can calculate the dimension of the set of solutions of (1) (being a polyhedral complex) with the complexity which depends polynomially on $n, m$ ?

Acknowledgements. The author is grateful to the Max-Planck Institut für Mathematik, Bonn for its hospitality during writing this paper and to Thorsten Theobald for valuable discussions.

\section{References}

[1] M. Akian, S. Gaubert, A. Guterman, The correspondence between tropical convexity and mean payoff games, Proc. 19 Intern. Symp. Math. Theory of Networks and Systems, Budapest (2010), 1295-1302.

[2] M. Bezem, R. Nieuwenhuis, E. Rodriguez Carbonell, Exponential behaviour of the Butkovic-Zimmermann algorithm for solving two-sided linear systems in max-algebra, Discrete Appl. Math. 156 (2008), 3506-3509.

[3] T. Bogart, A. N. Jensen, D. Speyer, B. Sturmfels, R. R. Thomas, Computing tropical varieties, J. Symb. Comput. 42 (2007), 54-73.

[4] P. Butkovic, Max-linear systems: theory and algorithms, Springer, 2010. 
[5] P. Butkovic, F. Hevery, A condition for the strong regularity of matrices in the minimax algebra, Discr. Appl. Math. 11 (1985), 209-222.

[6] P. Butkovic, K. Zimmermann, A strongly polynomial algorithm for solving two-sided linear systems in max-algebra, Discrete Appl. Math. 154 (2006), 437-446.

[7] M. Develin, F. Santos, B. Sturmfels, On the rank of a tropical matrix, In Combinatorial and computational geometry, Math. Sci. Res. Inst. Publ. 52, Cambridge Univ. Press, Cambridge (2005), 213-242.

[8] D. Grigoriev, Multiplicative complexity of a bilinear form over a commutative ring, Lect. Notes Comput. Sci. 118 (1981), 281-286.

[9] Z. Izhakian, L. Rowen, The tropical rank of a tropical matrix, Communic. Algebra 37 (2009), 3912-3927.

[10] K. H. Kim, F. W. Roush, Factorization of polynomials in one variable over the tropical semiring, arXiv:math/050116/v2.

[11] K. H. Kim, F. W. Roush, Kapranov rank vs. tropical rank, Proc. Amer. Math. Soc. 134 (2006), 2487-2494.

[12] T. Theobald, On the frontiers of polynomial computations in tropical geometry, J. Symbolic Comput. 41 (2006), 1360-1375. 